\title{
Trojans in Exosystems with Two Massive Planets
}

\author{
Rudolf Dvorak $^{1}$, Li-Yong Zhou ${ }^{2}$ and Helmut Baudisch ${ }^{1}$ \\ ${ }^{1}$ Universitätssternwarte, University of Vienna, \\ Türkenschanzsstrasse 17, A-1180 Vienna, AUSTRIA \\ email: dvorak@astro.univie.ac.at \\ ${ }^{2}$ Astronomy Department \& Key Laboratory of Modern Astronomy and Astrophysics in \\ Ministry of Education, Nanjing University, Nanjing 210093, CHINA \\ email: zhouly@nju.edu.cn
}

\begin{abstract}
We take as dynamical model for extrasolar planetary systems a central star like our Sun and two giant planets $m_{1}$ and $m_{2}$ like Jupiter and Saturn. We change the mass ratio $\mu=m_{2} / m_{1}$ of the two large planets for a wide range of $1 / 16<\mu<16$. We also change the ratio between the initial semi-major axes $\left(\nu=a_{2} / a_{1}\right)$ in the range of $1.2<\nu<3$ to model the different architecture of extrasolar planetary systems hosting two giant planets. The results for possible Trojans (Trojan planets) in the equilateral equilibrium points of the inner planet $m_{1}$ and the outer planet $m_{2}$ were derived with the aid of numerical integration. It turned out that in many configurations - depending on the mass ratios $\mu$ and the semi-major axes ratio $\nu$ - giant planets may host Trojans.
\end{abstract}

Keywords. Trojans, extrasolar planets, two massive planets

\section{Introduction}

The motion of celestial bodies around the stable Lagrangian points $L_{4}$ and $L_{5}$ (preceding and trailing equilibrium points) of planets in our Solar System (SS) is an interesting problem for Celestial Mechanics. The discovery of the first asteroid (later named Achilles) in libration around $L_{4}$ of Jupiter is due to the astronomer Max Wolf in Heidelberg. Ever since a great number of asteroids were observed and nowadays several thousand discoveries of such objects in the 1:1 Mean Motion Resonance (MMR) with Jupiter have been made.

The largest planet in the solar system is not the only planet that hosts asteroids along its orbit. Neptune also has such companions (up to now, 9 are known) and so do the terrestrial planets, Mars and Earth. In these stable equilibrium points, the centrifugal force on a third (massless) object finds equilibrium with the combined gravitational forces from the Sun and a massive planet (Fig. 1). This configuration of an equilateral triangle can also be stable when all three objects are massive. This was already known by Joseph Louis Lagrange (1736 - 1813) who theoretically investigated this problem without knowing its importance for future astronomy and especially for space astronomy. The socalled collinear equilibrium points $L_{1}, L_{2}$ and $L_{3}$ - also co-orbiting with the planet although in principle unstable, are now populated by many spacecrafts in librational 3dimensional orbits around them. The $L_{4}$ and $L_{5}$ Lagrangian points are located $60^{\circ}$ ahead of and $60^{\circ}$ behind the planet in the 1:1 MMR. Those points are stable for all planets in the Solar System and fulfill the stability condition primaries $m_{\text {planet }} / m_{\text {sun }}<1 / 27$. All asteroids close to $L_{4}$ and $L_{5}$ of Jupiter are named Trojans after mythological warriors from the Trojan War. As mentioned, the number of confirmed Trojans in the Solar System is steadily growing and as of today we know of Jupiter to have 3394 asteroids at $L_{4}$ and 
1811 at $L_{5}$, Neptune to have 6 Trojan asteroids at $L_{4}$ and 3 at $L_{5}$, Mars to have 1 Trojan at $L_{4}$ and 3 at $L_{5}$, and Earth to have 1 asteroid at $L_{4}$.

There exist many studies regarding Trojans in our Solar System (e.g., Mikkola et al. (1992), Robutel et al. (1995), Zhou et al. (2009), Zhou et al. (2011), Dvorak et al. (2012)). It is remarkable that the second largest planet Saturn does not host such bodies, and also Mercury, Venus and Uranus seem be without such companions (e.g., Nesvorný and Dones (2002)). In a recent study in different dynamical models, it turned out that the reason for the nonexistence of Saturn Trojans are the strong perturbations of Jupiter (Baudisch et al. (2012)).

As we now have knowledge of more than 800 extrasolar planets around other stars (see http://www.exoplanet.eu) where most of them are comparable with the size of Jupiter, an obvious question is to ask about possible Trojans (more precisely of terrestrial like planets) in 1:1 MMR with such planets. Especially large planets in a habitable zone around a star are subject of such investigations. The answer is relatively straightforward to give (Dvorak et al. (2004), Schwarz et al. (2004), Érdi and Sándor (2005), Schwarz et al. (2009)) when we just look at a single planet. As shown in our Solar System (Jupiter hosts Trojans, Saturn does not!) it is not so easy to deal with this problem when two massive planets are involved. The goal of this work is to find out the architecture of a planetary system with two giant planets such that it can accommodate a terrestrial like planet in a Trojan configuration.

The paper is divided into the following sections: first we explain our methodology, second we show the results in the respective stability plots for a larger outer planet $\mu /$ geq 1 , third we present the corresponding results for $\mu \leqslant 1$, and finally we will outline the next steps of this study, which consist primarily of determining the resonance involved to understand the structure of the initial condition diagrams and the probability of finding such interesting planets in the future.

\section{The method}

Using numerical integrations in a dynamical model of Star-Planet1-Planet2, we studied the stability of the Trojan regions of the two planets. We considered a Trojan to be a putative terrestrial planet and assumed that compared to the heavy gas giants, they can be considered to be massless. Given the symmetry of the preceding and trailing Lagrange points, we concentrated on the equilibrium point $L_{4}$ of the inner planet with mass $m_{1}$, and $L_{4}$ of the outer planet $m_{2}$. The initial positions for 100 fictitious Trojans were taken along the connecting line star- $L_{4}$ with a certain range in the semimajor axes $a$ for the Trojans around $L_{4}$. (see Fig. 1 ).

The initial difference in true longitudes of the two planets was set to $139^{\circ}$, but we also made test computation for a difference of $39^{\circ}$. The results were nearly identical. To ensure that the primary planets' stability does not affect the dynamics of the trojans, we tested the respective three body problem of star plus the two planets alone. We show the unstable region in Fig. 2 where we plotted the mass of $m_{2}$ (in this plot the larger mass) versus the initial semimajor axes $a_{2}$. The unstable region turned out to be not so quite different from analytical estimations up to large masses of the outer planet (thick red points in Fig. 2; after Gladman (1993).

We choose the following initial conditions for the two gas giants and the fictitious massless Trojans. Note that the mass of the central star and the semi-major axis of $m_{1}$ were set to unity:

- $m_{1}=0.001, a_{1}=1$

- $m_{1}<m_{2}<16 \cdot m_{1}$ with $\Delta m_{2}=1$ 


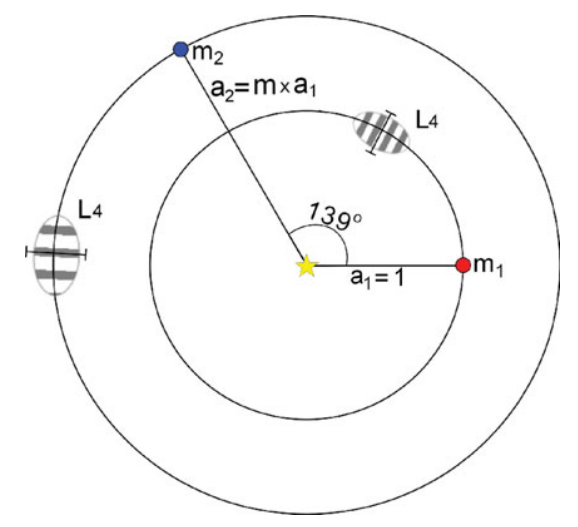

Figure 1. Schematic view of the initial configuration Star- $m_{1}-m_{2}$ and both $L_{4}$ Trojan regions.

- $m_{1}>m_{2}>\frac{m_{1}}{l}, l=1,2, \cdots, 16$

- $1.5 \cdot a_{1}<a_{2}<3 \cdot a_{1}$, with $\Delta a_{2}=0.01$

- $0.9 \cdot a<a_{\text {trojan }}<1.1 \cdot a$ for 100 massless bodies

With these initial conditions for 100 Trojans for 25 different mass ratios $\mu$ and different values for $a_{2}$ (we do not show the results for $a_{2}>2.2$ because the Trojan regions for that range were all stable) we carried out numerical integrations in the Newtonian framework for 1 million years. The integration method was the already well-tested Lie-integrator with an automatic step-size control, which in former studies turned out to be quite efficient compared to other methods (Hanslmeier and Dvorak (1984), Delva (1984), Eggl \& Dvorak(2010)). We emphasize that we just integrated the planar problem and have given the two primaries the initial osculating elements for Jupiter and Saturn with the exception for $a_{2}$ and the difference in the mean longitudes. To test the stability of an orbit, we have chosen a straight forward measure of the eccentricity of a Trojan along its orbit. In all former investigations, an eccentricity of $e>0.3$ in the future dynamical evolution of a Trojan led to an escape from the Trojan region.

\section{The results}

With the above initial conditions for the different dynamical models, we determined the orbital stability of Trojans (terrestrial planets) for different mass ratios $\mu$ of gas giants. At the same time, we also exmained the effect of an increase in the distance between the two planets independent of $\mu$.

\subsection{The $L_{4}$ region for $\mu>1$}

We first increased the mass of $m_{2}$ to $m_{2}=16 m_{1}$. We show the results in Figs. 3,4 and 5 for 3 selected masses, namely $m_{2}=1,8$, and $16 m_{1}$, respectively. We plot along the $x$-axis the cut through $L_{4}$ in the direction to the star with smaller and larger initial semimajor axis than the planet (see Fig. 1) and along the $y$-axes the initial semimajor axis of the second planet. As already mentioned, the largest eccentricity during the integration (color code), serves as a stability criterion. Dark colors stand for very small eccentricities and thus for stable orbits. Comparing the three figures on the left (inner $L_{4}$ region) and on the right side (outer $L_{4}$ region), it is clear that the large size of the stable region diminishes with a larger disturbing mass of $m_{2}$. At the same time, with initial larger separation of the two masses, the Trojans in these regions suffers less and less from their 


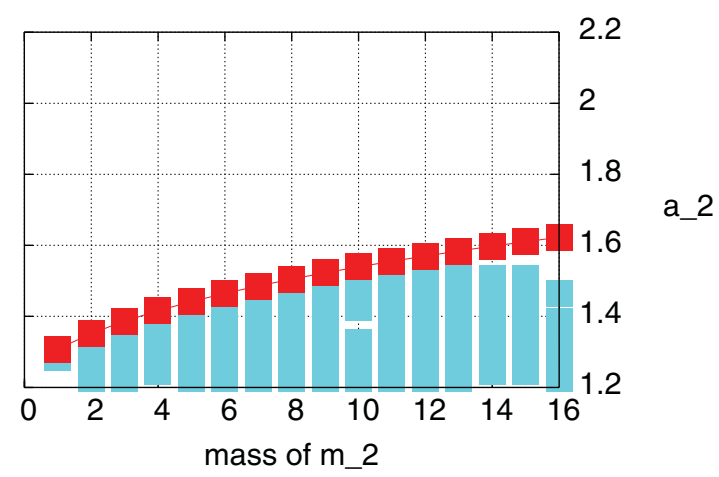

Figure 2. Stability region (upper white area) for the 3-body problem of a star and two planets: semi-major axis of the outer planet $\left(a_{2}\right)$ versus its mass $\left(m_{2}\right.$ in multiples of $\left.m_{1}\right)$. Blue points stand for unstable orbits, the red points are plotted after a simple analytical estimate
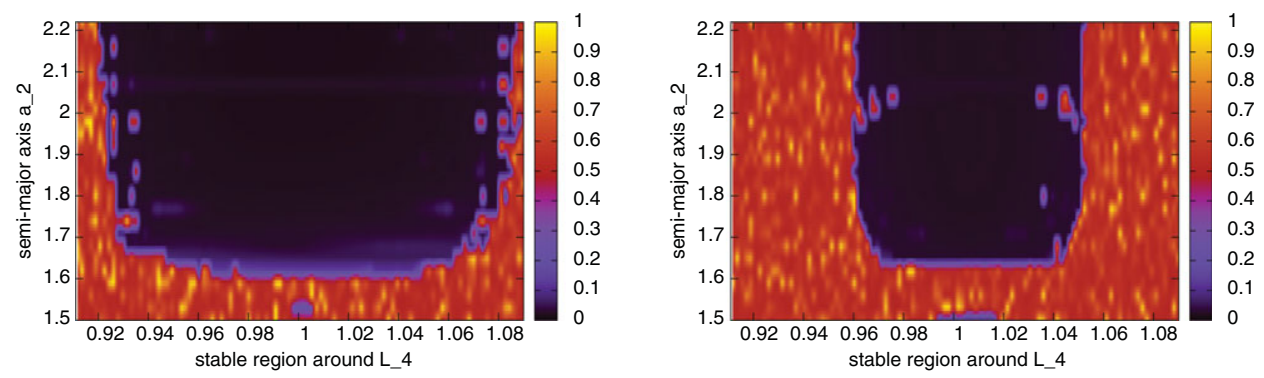

Figure 3. Stable region around the 'inner' equilibrium point $L_{4}$ of $m_{1}$ (left graph) and for the stable regions around the 'outer' equilibrium point $L_{4}$ of $m_{2}$ (right graph) for $m_{2}=m_{1}$.

perturbations, such that for $a_{2}>2.2$, a large fraction of Trojans close to $L_{4}$ survive. The two characteristic unstable stripes (visible in Figs. 4 and 5 left and right graphs) can be identified with the 2:1 and the 3:1 MMR between the Trojans located in that region, and the planet $m_{2}$ (Figs. 4 and 5 left graphs; inner $L_{4}$ region) or the planet $m_{1}$ (Figs. 4 and 5 right graphs; outer $L_{4}$ region) respectively. For $m_{2}=16 m_{1}$, only a small fraction of Trojans survive especially for the inner $L_{4}$ region. Note that in the case of equal-mass planets, the 'outer' stable $L_{4}$ region seems to be significantly smaller than the inner one. This is due to the fact that for the outer region, we used normalized semi-major axes for the cut through $L_{4}$.

We summarize the results in Fig. 6 where we plotted the surviving orbits (color code) for a given mass ratio $(\mu)$ (mass $m_{2}$ on the $x$-axes) and for a fixed value of $a_{2}$ ( $y$ axes). The regions at the bottom show that no Trojans survive up to $a_{2}=1.6$ in both experiments. This is quite a different result as expected from the test computations for the three body system star-planet1-planet2 in Fig. 2 for masses $m_{2}$ up to $m_{2}=6 \cdot m_{1}$. Even close-by planets survive for $a_{2} \sim 1.3$ and $a_{2} \sim 1.5$ depending on the mass of the second planet $m_{2}$. This means that additional second order resonances deplete the Trojan region (see conclusion). The band of unstable Trojan orbits in both regions due to the strong 3:1 resonance is also a characteristic of these systems. We should also mention that very probably the two vertical unstable stripes (Fig. 6 right graph) are due to secular interactions between the two planets. 

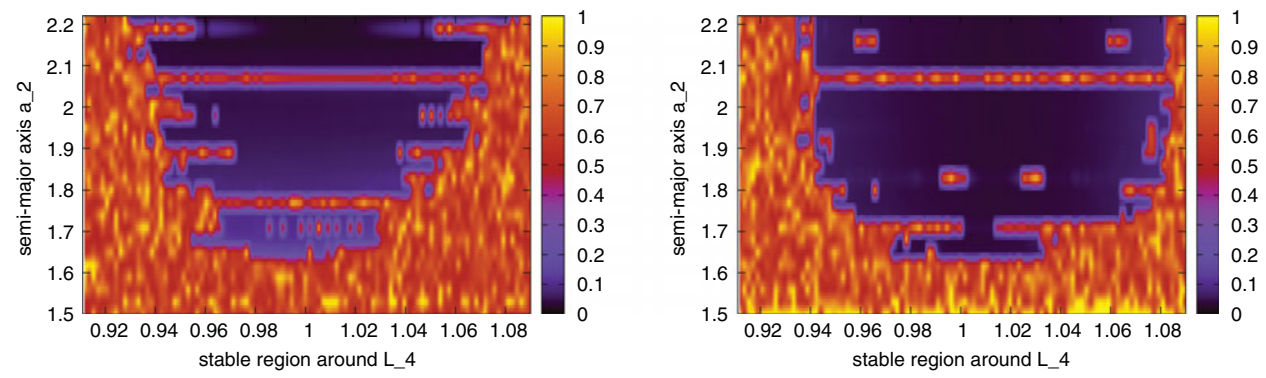

Figure 4. Captions like in 3 for $m_{2}=8 \cdot m_{1}$.
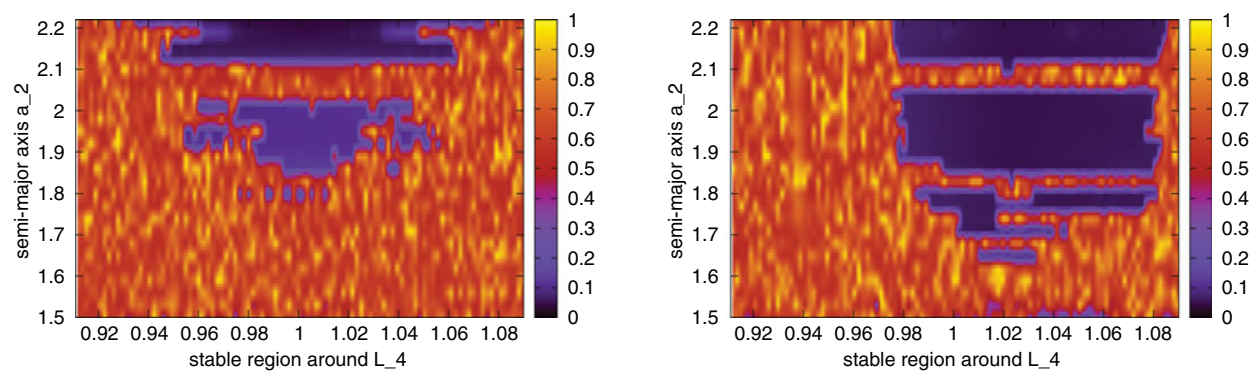

Figure 5. Captions like in 3 for $m_{2}=16 \cdot m_{1}$.
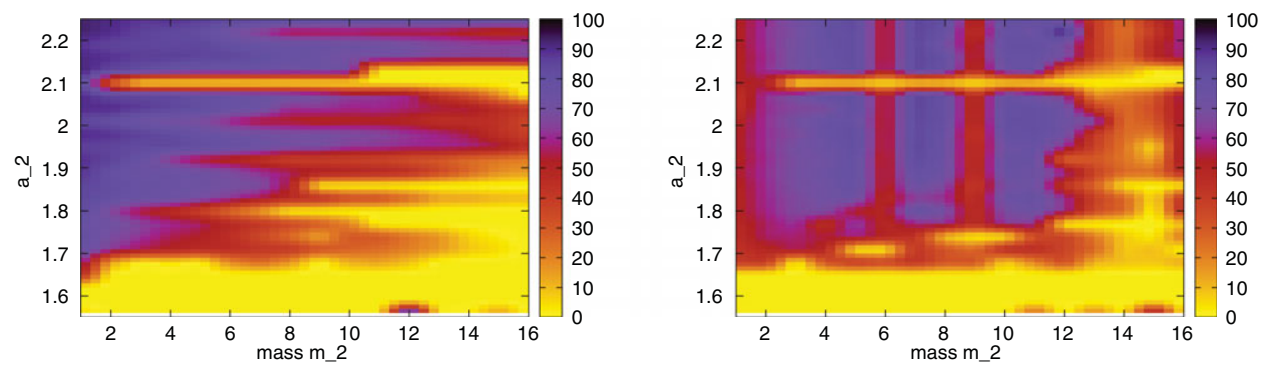

Figure 6. Global stability region around $L_{4}$ for different masses of $m_{2}$ (x-axes) versus the semimajor axes of $m_{2}$ for the inner $L_{4}$ equilibrium point (left graph) and the outer $L_{4}$ equilibrium point (right graph); the color stands for the number of stable orbits out of 100 (yellow means no Trojan is on a stable orbit.

\subsection{The $L_{4}$ region for $\mu<1$}

For smaller values of the mass of the outer planet, the inner stable regions at $L_{4}$ are very large (Fig. 7; left graph) and they increase continuously by decreasing $m_{2}$ (not shown in detail). In the outer regions, the stable regions resist the perturbation of $m_{1}$. The difference to the former results $(\mu>1)$ is that no 3:1 MMR and 2:1 MMR unstable stripes were found. Fig. 8 summarizes these results in particular the large stable regions for the inner $L_{4}$ (left graph).

Using the the results considering the gas giants to be Jupiter and Saturn, we can see from Fig. 8 (right graph, $m_{2}=1 / 3, a_{2} \sim 2$ ) that not many of the Trojans are stable for more than 1 million years. This result agrees in principle with a former study by Baudisch et al. (2012). We were not, however, able to confirm in our more global 

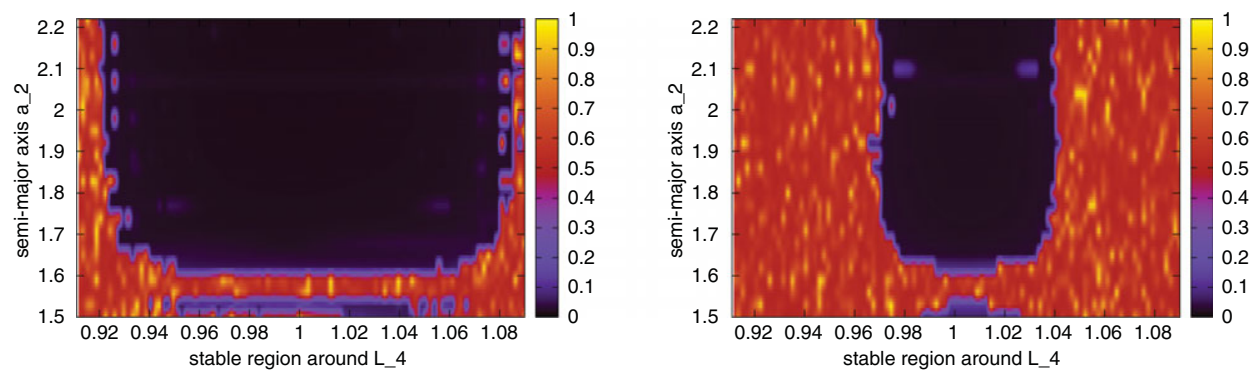

Figure 7. Stability region of $m_{1}\left(m_{2}=m_{1} / 2\right)$ around $L_{4}$; captions like in Fig. 3.
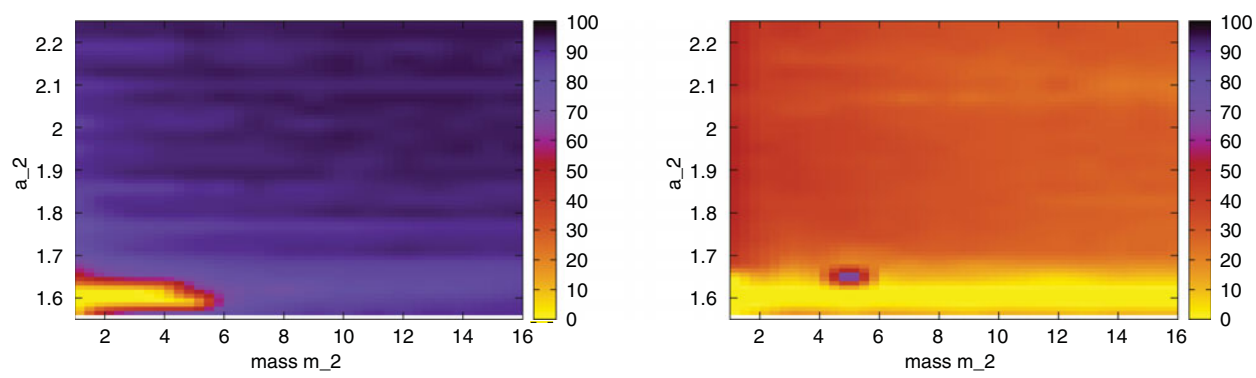

Figure 8. Global stability region around $L_{4}$ for different - smaller - masses $m_{2}$ (note that the labels are to be taken as $1 / m_{2}$ ) versus the semi-major axes of $m_{2}$; the color stands for the number of stable orbits out of 100 (yellow means no Trojan is on a stable orbit).

investigation that the Saturn Trojans suffer from an immediate depletion around the Lagrange point whereas a ring of orbits around this region remains stable for up to $10^{8}$ years.

\section{Conclusions}

To study the stability of Trojans, we integrated a large number of fictitious massless bodies close to the Lagrange point $L_{4}$ of two large planets similar to Jupiter or Saturn. The results showed that the regions of the 1:1 MMR with one of the planets are relatively stable when the outer planet is small compared to the inner one. Globally no Trojan can survive in the region where the difference of the semi-major axes is smaller than $\nu<1.6$. For values of $\nu>2.1$, the influence of the planets on the other Trojan area is very small, and we expect Trojans (Trojan planets) to be able to survive there. This is of great interest when the gas giant itself moves in the habitable zone around its host star. One next step in these studies is to carry out a detailed analysis of the frequencies, and the MMR and secular resonances responsible for the structure of the regions. Another very important study would be to use real data (obtained from the observation of exoplanetary systems) with two large planets. planets.

\section{Acknowledgements}

RD thanks the Austrian Science Foundation (Fonds zur Förderung der Wissenschaft, project S 11603-N16) for travel support. LYZ thanks supports from the National Basic Research Program of China (2013CB834904) and the Natural Science Foundation of China under grants No. 10833001 and No. 11073012. 


\section{References}

Baudisch, H. \& Dvorak, R. Where are the Saturn Trojans?, in Proceedings of the Journées 2011, "Systèmes de référence spatio-temporels", eds. H. Schuh, S. Böhm, T. Nilsson and N. Capitaine, Vienna University of Technology, pp. 225-228 (2012)

Beaugé, C., Sándor, Zs., Érdi, B., \& Süli, Á., Co-orbital terrestrial planets in exoplanetary systems: a formation scenario, A\&A 463, 359 (2007)

Delva, M., Integration of the elliptic restricted three-body problem with Lie series, Celest. Mech. 34, 145 (1984)

Dvorak, R., Pilat-Lohinger, E., Schwarz, R., \& Freistetter, F., Extrasolar Trojan planets close to habitable zones, A\&SA 426, L37 (2004)

Dvorak, R., \& Schwarz, R. On the stability regions of the Trojan asteroids, Celest. Mech. Dyn. Astr. 92, 19 (2005)

Dvorak, R., Lhotka, C., \& Zhou, L. The orbit of 2010 TK7: possible regions of stability for other Earth Trojan asteroids, A\&A $\mathbf{5 4 1} 127$ (2012)

Eggl, S. \& Dvorak, R., Lecture Notes in Physics, 790, 431 (2010)

Érdi, B. \& Sándor, Z., Stability of Co-Orbital Motion in Exoplanetary Systems, Celest. Mech. Dyn. Astr. 92, 113 (2005)

Gladman, B. Dynamics of systems of two close planets. Icarus, 106, 247 (1993)

Hanslmeier, A. \& Dvorak, R., Numerical Integration with Lie Series, A\&A 132, 203 (1984)

Mikkola, S. \& Innanen, K., A numerical exploration of the evolution of Trojan-type asteroidal orbits, AJ 104, 1641 (1992)

Nesvorný, D. \& Dones, L., How Long-Lived Are the Hypothetical Trojan Populations of Saturn, Uranus, and Neptune?, Icarus, 160, 271 (2002)

Robutel, P., Gabern, F., \& Jorba, A., The observed Trojans and the global dynamics around the Lagrangian points of the Sun Jupiter System, Celest. Mech. Dyn. Astr. 92, 53 (2005)

Schwarz, R., Gyergyovits, M., \& Dvorak, R., On the stability of high inclined L4 and L5 Trojans,Celest. Mech. Dyn. Astr. 90, 139 (2004)

Schwarz, R., Süli, Á., Dvorak, R., \& Pilat-Lohinger, E., Stability of Trojan planets in multiplanetary systems, Celest. Mech. Dyn. Astr. 104, 69 (2009)

Zhou, L. Y., Dvorak, R., \& Sun, Y. S., The dynamics of Neptune Trojan - I. The inclined orbits, MNRAS, 398, 1217 (2009)

Zhou, L. Y., Dvorak, R., \& Sun, Y. S., The dynamics of Neptune Trojans - II. Eccentric orbits and observed objects, MNRAS 410, 1849 (2011) 